Sir,

\section{Tattoo-associated uveitis}

We report a case of panuveitis and macular oedema associated with tattoo inflammation. The course of uveitis, which has responded to systemic immunosuppression, has followed the course of tattoo inflammation.

\section{Case report}

A 49-year-old man with known asthma, psoriasis and hypertension presented with bilateral reduced vision and floaters. Systematic enquiry revealed a chronic cough and tattoo swelling.

Visual acuities were 6/9 in the right eye and 6/18 in the left eye. Examination revealed severe bilateral panuveitis, multiple mid-peripheral atrophic punchedout fundal lesions, and bilateral cystoid macular oedema. His tattoos were swollen and raised (Figure 1a). Investigations for causes of uveitis, including serum ACE, chest X-ray, and CT scan were normal. A tattoo
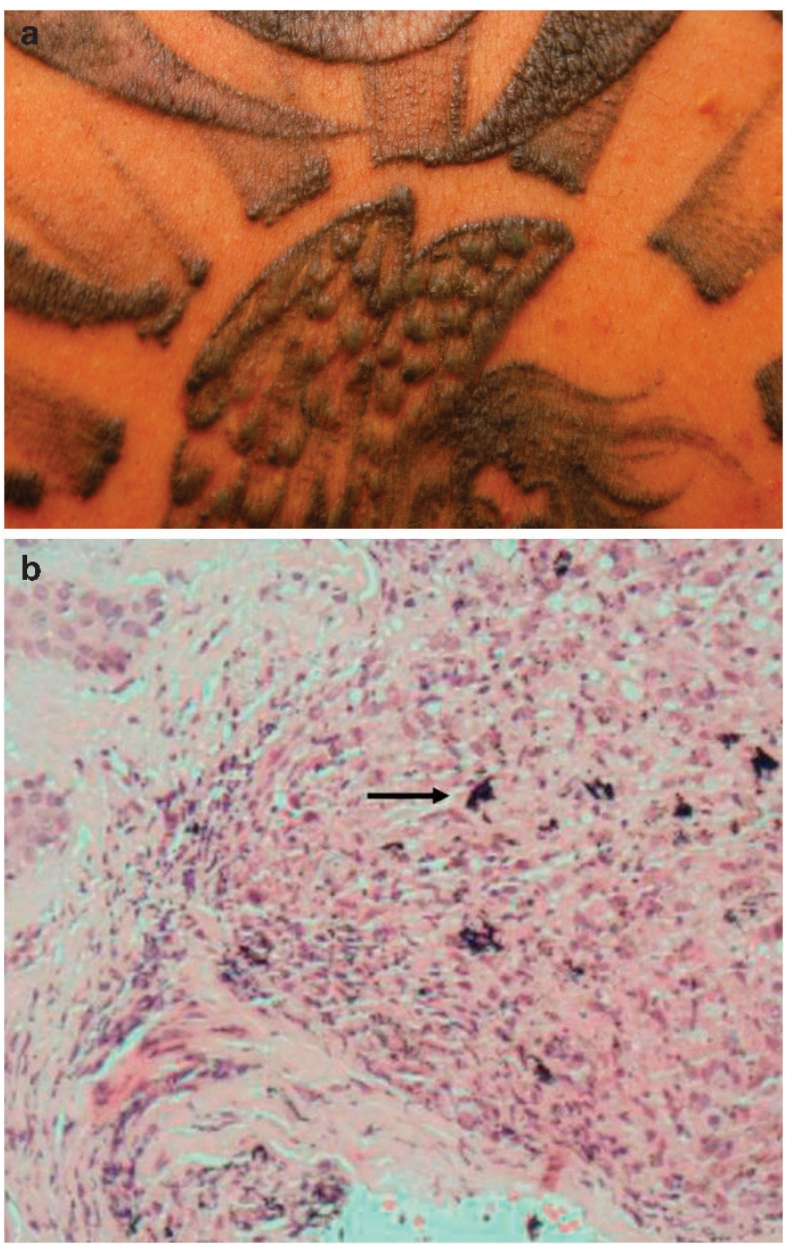

Figure 1 (a) Photograph showing the swollen, elevated tattoo. (b) Histological section of skin biopsy from inflamed tattoo. The non-caseating granulomas appear to be formed around included tattoo pigment granules (arrow). The histological appearances of a foreign body granuloma and sarcoidosis are very similar, and it is impossible to exclude sarcoidosis on histology of such a granuloma. biopsy revealed a florid granulomatous response around the included tattoo pigment (Figure 1b).

The uveitis and macular oedema resolved with oral prednisolone $75 \mathrm{mg}$ OD, which also reduced the tattoo swelling. Vision improved to $6 / 6$ bilaterally. After 4 months, following steroid tapering, his uveitis recurred and azathioprine was started. Systemic immunosuppression with prednisolone and azathioprine has controlled the uveitis to date.

\section{Comment}

Tattoo granulomas can occur in sarcoidosis but may also be a delayed allergic reaction to dyes used in tattoos. ${ }^{1}$ Similar cases of uveitis associated with tattoos have been described and in three cases the uveitis resolved after tattoo excision. ${ }^{1-3}$ All cases initially required systemic immunosuppression to control the uveitis.

The inclusion of tattoo pigment in granulomas suggests that these foreign bodies initiate an inflammatory response and activate lymphocytes. In this case the timing and course of tattoo inflammation has mirrored the ocular inflammation. Underlying sarcoidosis is a possibility, but there are no other clinical, laboratory, or radiological features, particularly no skin disease away from his tattoos. The presence of extensive foreign body tattoo pigments, which are a complex mix of heavy metals, dyes, and organic compounds, ${ }^{4}$ may have contributed to his immune dysregulation. As the tattoos are very extensive, excision is not an option in this case.

This case illustrates an unusual association with uveitis. It is important to note that tattoo-associated uveitis can occur without evidence of sarcoidosis. Ophthalmologists should ask about inflamed tattoos in their systematic history of patients with uveitis, particularly in elusive cases. Treatment should be according to standard therapy for non-infectious uveitis, but the condition of the tattoos can assist with monitoring treatment.

\section{Conflict of interest}

The authors declare no conflict of interest.

\section{References}

1 Rorsman H, Brehmer-Andersson E, Dahlquist I, Ehinger B, Jacobsson S, Linell F et al. Tattoo granuloma and uveitis. Lancet 1969; 2(7610): 27-28.

2 Barabasi Z, Kiss E, Balaton G, Vajo Z. Cutaneous granuloma and uveitis caused by a tattoo. Wien Klin Wochenschr 2008; 120(1-2): 18.

3 Moschos MM, Guex-Crosier Y. Retinal vasculitis and cystoid macular edema after body tattooing: a case report. Klin Monatsbl Augenheilkd 2004; 221(5): 424-426.

4 Helmenstine AM. Tattoo ink chemistry. (web 9 November 2009).

N Saliba, ME Owen and N Beare

St Paul's Eye Unit, Royal Liverpool University

Hospital, Liverpool, UK

E-mail: nax024@gmail.com

Eye (2010) 24, 1406; doi:10.1038/eye.2010.17; published online 26 February 2010 